Revue internationale P.M.E.

Économie et gestion de la petite et moyenne entreprise

Revue

internationale

PME

\title{
Milieux innovateurs: concept et application
}

\section{Marc-Urbain Proulx}

Volume 7, numéro 1, 1994

URI : https://id.erudit.org/iderudit/1008370ar

DOI : https://doi.org/10.7202/1008370ar

Aller au sommaire du numéro

Éditeur(s)

Presses de l’Université du Québec

ISSN

0776-5436 (imprimé)

1918-9699 (numérique)

Découvrir la revue

Citer cet article

Proulx, M.-U. (1994). Milieux innovateurs: concept et application. Revue internationale P.M.E., 7(1), 63-84. https://doi.org/10.7202/1008370ar

\section{Résumé de l'article}

Ce texte se divise en deux parties. Nous proposons dans un premier temps, une synthèse des composantes induites par le concept de "milieu innovateur". Tous les facteurs répertoriés dans la littérature sont exposés dans un modèle général. Selon le corpus théorique actuel, les décideurs (ou acteurs) du milieu local ou régional doivent chercher collectivement la combinaison appropriée de facteurs nécessaires pour leur propre territoire. Ainsi, dans un deuxième temps, une procédure de planification est offerte aux territoires en quête d'innovation et de dynamisme socio-économique. Ensuite, nous exposons les tâches à effectuer aux différentes étapes d'organisation d'un milieu innovateur. De fait, la procédure de planification proposée prend la forme d'un processus systématique d'apprentissage continu pour les acteurs de la collectivité territoriale. 


\title{
Milieux innovateurs: concept et application
}

\author{
Marc-Urbain PROULX* \\ Université du Québec à Chicoutimi
}

\begin{abstract}
RÉSUMÉ
Ce texte se divise en deux parties. Nous proposons dans un premier temps, une synthèse des composantes induites par le concept de "milieu innovateur". Tous les facteurs répertoriés dans la littérature sont exposés dans un modèle général. Selon le corpus théorique actuel, les décideurs (ou acteurs) du milieu local ou régional doivent chercher collectivement la combinaison appropriée de facteurs nécessaires pour leur propre territoire. Ainsi, dans un deuxième temps, une procédure de planification est offerte aux territoires en quête d'innovation et de dynamisme socio-économique. Ensuite, nous exposons les tâches à effectuer aux différentes étapes d'organisation d'un milieu innovateur. De fait, la procédure de planification proposée prend la forme d'un processus systématique d'apprentissage continu pour les acteurs de la collectivité territoriale.
\end{abstract}

\begin{abstract}
Our contribution is divided in two parts. We propose first a synthesis of the theoritical components induced by the concept of "innovative environment". All the factors of the literature are considered in a global model. According to the theory, the local or regional deciders (actors) must find collectively the optimal combination of factors for their own territory. Then, in the other part, a planning procedure is offered to the territories in quest of innovation and socio-economic dynamic. In that sense we expose the tasks in different steps towards the organization of an innovative environment. In fact the planning procedure takes the form of a systematic continuous learning process for the deciders of the territorial collectivity.
\end{abstract}

* Marc-Urbain Proulx enseigne à l'Université du Québec à Chicoutimi où il fait par ailleurs partie du Groupe de recherche et d'intervention régionales. Il est membre de plusieurs associations scientifiques des deux côtés de l'Atlantique. En 1992, il fut invité, par la Revue Canadienne des Sciences Régionales, à diriger un numéro thématique sur les «milieux innovateurs». Adresse: Département des sciences économiques et administratives, Université du Québec à Chicoutimi, 555, boul. de l'Université, Chicoutimi (Québec) G7H 2B1. 


\section{RESUMEN}

Este texto se divide en dos partes. En primer lugar, proponemos una síntesis de los componentes inducidos en el concepto "medio innovador". Todos los factores catalogados en la literatura aparecen según un modelo general. Según el corpus teórico actual, los dirigentes (o actores) del territorio deben buscar colectivamente la combinacion optima de los factores necesarios para su territorio. Además, en una segunda parte, ofrecemos un procedimiento de planificacion de los territorios locales y regionales en busca de innovacion y de dinamismo socio-económico. Ante esta situacion, exponemos las diferentes tareas que hay que efectuar en las diferentes etapas de organización de un medio innovador. De hecho, el procedimiento de planificación expuesto toma la forma de un proceso sistemático de aprendizaje continuo para los actores de la colectividad territorial.

\section{Introduction}

Bien que le vocable «milieu» soit utilisé depuis longtemps par les sciences naturelles et par la discipline du développement communautaire, plusieurs analystes des diverses sciences sociales l'ont adopté au cours des deux dernières décennies. La notion en question s'est alors dotée d'éléments conceptuels multidisciplinaires fort pertinents. En corollaire, la recherche empirique a généré de nombreux faits qui permettent d'ancrer et de mesurer de multiples variables du milieu. Au tournant des années 1990, les résultats cumulés de la recherche, notamment par Aydalot (1986), Aydalot et Keeble (1988), RIPME (1989), Camagni (1991), RERU (1991), Benko et Lipietz (1992), Maillat et Perrin (1992), Ratti (1992), RCSR (1992), Maillat et al. (1993) et autres ouvrages du GREMI ${ }^{1}$, forment, avec les nombreuses monographies indépendantes, un corpus suffisamment important pour nous permettre d'avancer l'émergence inéluctable d'une théorie des milieux ${ }^{2}$ que l'on qualifie quelquefois de locaux, de régionaux, d'entrepreneuriat, d'incubateurs, de fertiles, etc., mais généralement d'innovateurs.

Dans la construction théorique, le nombre, la richesse et la diversité des contributions rendent ambitieuse toute démarche de synthèse. Néanmoins, les efforts en ce sens sont inévitablement bienvenus autant par les scientifiques et les académiciens que par les praticiens du développement socio-économique.

1. Le Groupe européen de recherche sur les milieux innovateurs a organisé plusieurs colloques sur diverses dimensions du sujet depuis 1984.

2. Il est à noter que certains chercheurs travaillent dans le même sens en utilisant des vocables différents tels que districts industriels, systèmes spatialisés de production, espaces de soutien, structures spatiales d'incubation, niches, etc. 
Une partie de ce texte s'inscrit dans cette optique d'un essai de synthèse du corpus théorique sur les milieux innovateurs. Les facteurs isolés dans la littérature, parce qu'influençant la qualité d'innovation du milieu, seront exposés selon un modèle général comprenant huit grandes composantes. Toutefois, nous ne nous attarderons sur aucune composante ou indicateur en particulier. Notre contribution spécifique concernera plutôt l'articulation d'une procédure générale de planification d'un milieu innovateur pour un territoire local ou régional.

\section{Circonstances favorables à la nouvelle théorie}

Il existe certes de nombreuses orientations pouvant être considérées importantes dans l'évolution récente des sciences sociales. En conséquence, la théorie des milieux innovateurs émerge actuellement dans le cadre d'un certain nombre de tendances lourdes. Notre lecture nous permet à cet égard de considérer sept grandes tendances circonstantielles qui favorisent l'élaboration de cette nouvelle théorie du développement.

\section{SCHÉMA 1}

\section{Circonstances favorables à la nouvelle théorie}

Le déclin des modes traditionnels

de régulation économique

Le paradigme du développement par le bas

La montée de l'approche systémique

La perspective méso-analytique

L'exemple des territoires à succès

Le phénomène PME

L'enjeu local de l'emploi

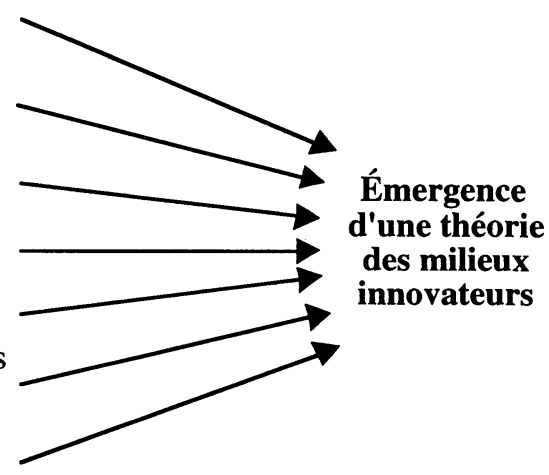

Étant donné le déclin du modèle fordiste de production-consommation de masse d'une part et, d'autre part, la crise financière de l'État interventionniste (keynésien), plusieurs analystes de l'économie contemporaine de plus en plus mondialisée cherchent la formule appropriée pour un nouveau mode de régulation socio-économique adapté aux enjeux actuels et aux nouvelles 
tendances d'avenir. Dans ce mouvement de recherche, un certain nombre de raisons ${ }^{3}$ sont des arguments pour plusieurs spécialistes en faveur du rôle primordial qui doit être joué par l'assise territoriale correspondant au milieu de vie de la population, des travailleurs et des organisations. Un resserrement communautaire à la base s'avère ainsi proposé afin de mieux concilier la turbulence des grands changements en cours actuellement. En ce sens, les arguments auxquels on fait le plus référence sont certes ceux de Piore et Sabel (1984) sur les qualités des formes spatiales de petites dimensions qu'ils appellent «districts» par référence aux travaux de Marshall (1890-1919). Selon cette conception marshallienne, la contiguïté des entreprises sur un espace donné (district/milieu) produit généralement des effets externes ${ }^{4}$ positifs à un certain degré. Le principal enjeu de la recherche sur les milieux innovateurs devient à l'évidence de réactualiser cet avantage comparatif dans le contexte contemporain.

La grande popularité de ce concept de milieu innovateur chez les analystes scientifiques n'est évidemment pas indépendante du retour en force du paradigme de développement par le bas depuis le tournant des années 1980 (Stohr et Taylor, 1981). C'est que les principales théories de développement régional par le haut ${ }^{5}$ furent particulièrement décevantes dans leur capacité de réduire convenablement les inégalités interterritoriales. On s'en remet ainsi largement aux forces et au dynamisme de la base (individus, organisations, communautés) pour créer les conditions de leur propre développement. Cette approche ascendante, noblement qualifiée par certains de néo-humanisme, devient la nouvelle orthodoxie qui oriente actuellement plusieurs écoles de recherche théorique et empirique. L'une des orientations majeures s'avère concernée par l'utilisation d'une approche globale d'analyse au niveau des communautés de base. En ce sens holistique, le développement n'est pas que mesuré en termes économiques, mais considéré aussi dans ses aspects sociaux, culturels, politiques et environnementaux, généralement perçus comme étant en amont de l'économique.

3. Affaiblissement du rôle de l'État; respect de la diversité socioculturelle; division locale de la production; flexibilité nécessaire de la production; redéploiement industriel.

4. Ces externalités de districts (atmosphère industrielle; culture technique; mobilité des compétences; échanges formels et informels ; professionnalité ambiante; etc.) seraient un atout important pour obtenir la flexibilité nécessaire actuellement dans la production industrielle.

5. Théorie de la base exportatrice; théorie de la localisation industrielle ; principe du processus cumulatif circulaire; théorie des pôles de croissance. 
Il faut aussi préciser que cette orientation vers une approche multidimensionnelle du milieu n'est pas indépendante de la perspective systémique qui a largement influencé les sciences sociales depuis Berthalanffy (1950). La théorie de l'organisation et la théorie de la firme en furent notamment enrichies profondément. Pour ces deux théories, le modèle classique d'analyse souvent qualifié de wébérien s'enrichit, en fait, des apports d'une approche alternative dite «environnementale». Cela permet de réfléchir bien au-delà de la traditionnelle mécanique d'efficacité interne et de considérer la micro-organisation tel un système ouvert et en constante interaction avec son macro-environnement dont elle est tributaire, d'ailleurs, dans sa quête d'intrants. À cet effet, puisque les processus décisionnels s'effectuent selon des démarches séquentielles courtes (Planque, 1988), notamment en ce qui concerne les décisions d'opération, l'environnement immédiat devient tout à fait essentiel sous l'angle de son contenu en intrants (ressources-compétences-information) nécessaires aux organisations.

Pour la science économique en général et l'économie régionale en particulier, cette focalisation directe sur l'espace-milieu s'inscrit dans une perspective dite méso-analytique. Étymologiquement, la mésologie s'avère l'étude des milieux de vie. En ce sens, il ne s'agit plus de spatialiser les outils disponibles (offre, demande, production, investissement, etc.) de manière indépendante selon la tradition, mais bien de partir des espaces-milieux et d'y appliquer systématiquement les outils micro-économiques et macro-économiques pour l'analyse rigoureuse des phénomènes observés. Cette perspective méso offre une opportunité singulière de renouvellement de l'économie régionale (Perrin, 1983-1992). C'est sûrement pourquoi la théorie émergente sur les milieux innovateurs occasionne actuellement, en science régionale, un si grand enthousiasme.

Cet enthousiasme théorique est d'ailleurs fort bien soutenu empiriquement. En effet, il fut largement démontré dans la plupart des pays occidentaux que certains milieux ${ }^{6}$ sont plus innovateurs et plus fertiles que d'autres sans répondre toutefois aux critères traditionnels et connus de croissance et de développement. L'observation et l'analyse de ces espaces, de ces districts, de ces vallées, de ces régions, de ces niches... permettent d'induire une panoplie de

6. Aux exemples prestigieux de la route 128 , du comté d'Orange et de SophiaAntipolis, on ajoute de multiples cas plus modestes, mais tout à fait convaincants tels que les districts de la troisième Italie, le bassin d'Alès, le milieu de Besançon, le pays d'Aix, le Jura suisse, le milieu de Cambridge (R.U.), la région de la Beauce, ainsi que les nombreuses petites «silicone valley» identifiées ici et là dans la plupart des pays développés ou en développement. 
formules gagnantes, souvent inédites, quelquefois inusitées et régulièrement transposables que très difficilement. Ces milieux innovateurs identifiés représentent un phénomène fort intéressant, car il s'inscrit à l'encontre de la théorie classique de l'innovation qui s'appuie largement sur le principe de diffusion à partir des grands centres urbains. Définie telle une nouvelle combinaison d'intrants, l'innovation ${ }^{7}$ apparaît alors pouvoir aussi émerger de manière endogène dans un espace périphérique donné. Il devient tout à fait essentiel d'en comprendre les rouages afin de multiplier le processus là où le besoin se fait sentir, notamment dans les espaces sous-développés des territoires nationaux.

La fertilité effective chez certains milieux et désirée chez d'autres est souvent mesurée en termes de petites et moyennes entreprises (PME). C'est que la PME est devenue depuis une vingtaine d'années un élément essentiel de la dynamique économique (Chicha et al., 1985) en créant la majorité des emplois dans les pays occidentaux. Il est largement soutenu que la PME ne répond pas aux facteurs d'attraction chers à la théorie de la localisation. D'où le postulat général de la recherche sur les milieux innovateurs qui stipule que les activités sont plutôt générées par leur environnement immédiat ou milieu local (Aydalot, 1984). À cet égard, la fertilité du milieu relève de sa capacité d'incuber et de faire croître des PME grâce aux conditions appropriées de son substrat vis-à-vis les nécessités des processus de production actuels et anticipés.

La création d'emplois par les petites initiatives innovatrices qui prennent assise dans leur contexte local fut d'ailleurs largement proposée, par les spécialistes, comme un enjeu pertinent de la politique économique. En utilisant la notion de «bassins locaux d'emplois» ou «marchés locaux pour l'emploi », la plupart des pays occidentaux ont lancé des programmes concernés par l'offre de travail (travailleurs) et spécifiquement adaptables aux différents contextes spatiaux sur les territoires nationaux (OCDE, 1982). Puisque ces interventions ont déjà généré des retombées considérables, notamment au plan de l'adaptation des compétences et de lancement d'initiatives qui valorisent le savoir-faire local (GREFFE, 1988), l'emploi est devenu un objectif qui justifie actuellement la décentralisation de responsabilités vers les entités territoriales de petite dimension. La pertinence du concept de milieu innovateur gagne alors encore un argument à sa défense.

Ainsi, cette argumentation reliée aux sept grandes tendances circonstantielles bien que décrites sommairement, nous fait comprendre tout l'intérêt scientifique que suscite, depuis une décennie, le phénomène des milieux innovateurs.

7. Nouvelles technologies; nouvelles méthodes de production ; nouveaux produits; amélioration de produits existants; nouvelles techniques de commercialisation; nouvelles pratiques de gestion; nouveaux marchés; etc. 


\section{Définition du milieu innovateur}

Dans la littérature, le concept «milieu» possède déjà un certain nombre de définitions convergentes. Bien qu'il s'agisse bel et bien de la qualité d'innovation sur un territoire, il faut tout de suite souligner que le milieu innovateur possède un contenu différent des concepts de parc industriel, de parc technologique, de parc scientifique et de technopole qui furent largement appliqués, à grand renfort de publicité et de ressources publiques, ces dernières années. Par rapport à ces concepts générés par la recherche sur les conditions spatiales à l'innovation, la première caractéristique typique du milieu concerne sa globalité puisqu'il incorpore non seulement les entreprises, mais aussi la population, les travailleurs, les multiples organisations ainsi que les multiples dimensions sociales et culturelles.

Considérant l'importance du corpus théorique en élaboration qui contient incidemment un grand nombre de variables mises en évidence, on ne peut que retenir une définition très large et englobante. Puisque le concept de district est souvent associé ou confondu avec celui de milieu, on réfère pertinemment à la définition de Becattini (1992) à savoir «une entité socio-territoriale caractérisée par la présence active d'une communauté de personnes et d'une population d'entreprises dans un espace géographique et historique donné». Si cette définition colle bien à la réalité du milieu telle qu'entendue par la littérature, elle n'insiste toutefois pas suffisamment sur le caractère innovateur. À cet égard, Maillat (1992) est plus précis en considérant que le milieu innovateur comprend cinq composantes soit: un espace géographique, une culture technique, un collectif d'acteurs, une logique d'organisation et une dynamique d'apprentissage. Nous pourrions citer, par ailleurs, plusieurs autres définitions dont la plupart convergent finalement dans le sens d'un «territoire dont les acteurs génèrent un processus collectif cognitif qui engendre un cadre, un climat, une atmosphère ou une culture au dynamisme social, politique, culturel, technologique, administratif et économique».

Ces définitions très larges montrent bien que nous sommes à l'aube d'une recherche qui n'a engendré jusqu'à maintenant que très peu de lois applicables d'une manière générale. Néanmoins, on s'entend largement à reconnaître que la qualité essentielle d'un milieu innovateur concerne la production collective, sur l'espace en question, d'une véritable synergie territoriale (Ratti, 1991). À cet égard, la question du comment fait l'objet de plusieurs hypothèses et de plusieurs monographies, mais demeure malgré tout, pour l'instant, ouverte. 


\section{Le modèle d'analyse du milieu innovateur}

Le milieu innovateur, c'est-à-dire créateur de nouvelles activités ${ }^{8}$ à caractère économique ${ }^{9}$, possède de toute évidence des dimensions spatiale, sociale, culturelle, environnementale, administrative et économique. Les nombreuses contributions recensées font ressortir, en somme, un très grand nombre de facteurs, nouveaux et anciens, qui influencent les qualités du milieu innovateur dans un territoire donné. Selon notre lecture, il devient possible de classer tous ces facteurs dans huit catégories qui deviennent en conséquence les grandes composantes des milieux innovateurs. Cette catégorisation nous permet dès lors de construire un modèle octogonal.

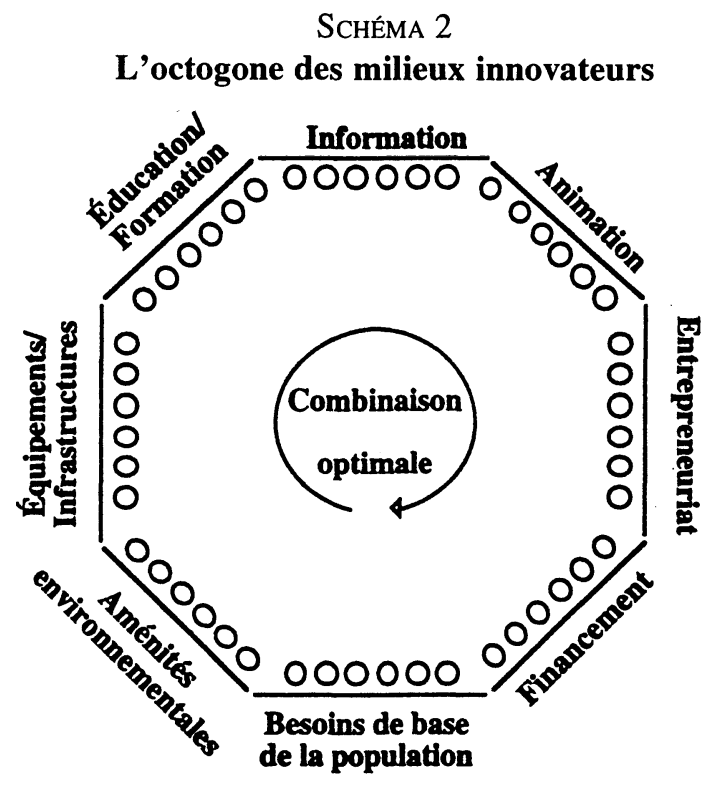

1. Satisfaction des besoins de base de la population: santé, éducation, nourriture, loisirs, habitation, sports, transport en commun, activités valorisantes, etc.

2. Présence d'aménités environnementales : parcs récréatifs, faible taux de pollution, qualité des éléments naturels, sports et loisirs de plein air, proximité entre travail et habitation, ensoleillement, etc.

8. De nature sociale, culturelle, administrative, forestière, agricole, industrielle, etc., qui génèrent un investissement, des emplois nouveaux ou de la valeur ajoutée.

9. Investissement, emplois, production ou valeur ajoutée. 
3. Équipements et infrastructures d'aménagement du territoire: transport et communication, terrains, bâtiments, aires industrielles, aires commerciales, hôpitaux, écoles, garderies, centres communautaires, centres de loisirs, centres culturels, centres d'entreprises, etc.

4. Éducation, formation et perfectionnement des compétences : maison d'enseignement, climat de travail, santé-sécurité au travail, culture technique, cercles d'apprentissage, cercles de qualité du travail, fidélité des travailleurs, mobilité des compétences, etc.

5. Accès à l'information: sur les opportunités, les concurrents, la conjoncture économique, les programmes publics, les modalités d'exportation, les brevets d'inventions, les réglementations, les technologies disponibles, les nouvelles techniques, etc.

6. Animation socio-économique: plans d'opportunités, services techniques, appuis aux initiatives, mise en communication d'acteurs et d'éventuels partenaires, événements culturels, valorisation des savoirfaire, etc.

7. Entrepreneuriat: climat d'affaires, culture entrepreneuriale, atmosphère industrielle, saine compétition, services d'aide à la gestion, formation des entrepreneurs, visibilité des modèles à succès, recrutement de jeunes entrepreneurs potentiels, etc.

8. Financement: capital de risque, programmes d'aide financière, attitudes des institutions financières, fonds populaires, services bancaires, etc.

Chez ces huit grandes catégories de facteurs, une panoplie d'organisations privées, publiques et collectives interviennent selon diverses logiques; chacune contribue à faire de son territoire local ou régional d'intervention un milieu innovateur.

Tous les facteurs ne sont pas également présents dans tous les milieux qualifiés d'innovateurs. De fait, notre synthèse nous permet de considérer que la combinaison optimale de facteurs afin qu'un territoire devienne «milieu innovateur» est, par essence ${ }^{10}$, différente d'un lieu à un autre. Il est largement avancé à cet effet que c'est au milieu lui-même de s'organiser par l'entremise de ses décideurs (organisations) et de créer les conditions sociales, culturelles, technologiques, administratives, politiques, économiques, etc. spécifiques et appropriées au dynamisme innovateur dans leurs activités actuelles ou potentielles. Il s'agit de trouver dans les huit grandes catégories de facteurs, la recette

10. En raison du fait que les ressources, la base économique, les potentialités, les besoins, les opportunités et les contraintes varient d'un territoire à l'autre. 
appropriée au territoire local ou régional en quête d'innovation et de développement. On en déduit ainsi que toutes les organisations reliées à ces multiples facteurs potentiels sont dès lors à mobiliser vers la recherche collective de la solution.

En effet, les expériences de «milieux à succès », de «milieux innovateurs» ou de territoires dotés de «l'effet milieu» font largement ressortir l'importance de cette démarche d'organisation collective du territoire. Par contre, les modalités opérationnelles sont de toute évidence nombreuses. Divers libellés existent dans la littérature pour désigner ce phénomène communautaire général et ses variantes: processus collectif cognitif (Perrin, 1983); réseaux interorganisationnels (Mulford, 1983) ; concertation (Stohr, 1985); coopération communautaire (Jessop et Weaver, 1987); activité résillière (Proulx, 1989); partenariat (Gagnon et Klein, 1991); systèmes de conventions territoriales (Planque, 1992); réseau de réseaux (Perrin, 1992). Quel que soit le qualificatif utilisé, l'une des pièces maîtresses dans la création d'un milieu innovateur concerne le processus collectif de collaboration interorganisationnelle sur une base volontaire et en respect de l'autonomie des divers acteurs.

On insiste généralement sur l'aspect volontaire du processus. C'est que les conditions de l'environnement organisationnel du territoire-milieu déjà institutionnellement occupé par diverses organisations autonomes sont incompatibles avec l'introduction d'un processus dirigiste et rigide d'intégration territoriale des actions par un pouvoir centralisateur quelconque. Par conséquent, la plupart des procédures d'intégration, d'organisation ou de planification territoriale préconisées actuellement dans la littérature s'appuient sur un cadre flexible, capable d'engendrer la collaboration volontaire des décideurs des multiples organisations qui interviennent sur le territoire. De cette collaboration dépend la capacité de générer des effets positifs. En ce sens, plusieurs spécialistes s'entendent pour lier la qualité de cette collaboration interorganisationnelle avec l'état de la culture territoriale (culture organisationnelle; culture de planification; culture de concertation) qui existe sur le territoire local et régional (Friedmann, 1987; Blakely, 1987; Perrin, 1992). La création d'un milieu innovateur, dynamique et capable de mettre en œuvre le développement local ou régional, doit être envisagée, en premier lieu, sous l'angle général de la culture territoriale dans laquelle baignent la population, les travailleurs et les organisations.

\section{Identité territoriale et culture territoriale}

Si la définition anthropologique de la culture nous réfère à un système structurant d'idées, de significations, de symboles ou de connaissances, la culture territoriale d'un éventuel milieu innovateur s'avère, à notre sens, un système structurant de processus décisionnels concernant la communauté territoriale. En 
ce sens, la culture joue son rôle dynamique et permet aux acteurs de s'exprimer, de libérer leur créativité ainsi que de se confronter aux autres acteurs (Bassand et al., 1985). Selon notre lecture, la composante organisationnelle de la culture sur un territoire local ou régional est associée à la capacité endogène des individus de prendre en main collectivement leur devenir communautaire et l'organisation d'un milieu de vie tel que désiré par et pour la collectivité.

La littérature scientifique nous renseigne sur les nombreux facteurs liés aux valeurs et aux comportements qui influencent de toute évidence cette capacité de prise en main communautaire. Il s'avère toutefois, par essence, fort difficile de mesurer la plupart de ces facteurs. Notre synthèse de la littérature nous permet de faire un premier essai d'inventaire des principaux facteurs organisationnels qui se retrouvent dans la culture territoriale d'un milieu.

- traditions à la coopération, à l'entraide et au partenariat ;

- capacité collective de consensus communautaire;

- respect et solidité de la solidarité communautaire;

- interdépendances et confrontations institutionnelles;

- corporatisme et alliances professionnelles;

- partisanerie politique / alliances stratégiques ;

- participation populaire aux processus décisionnels communautaires;

- présence de phénomènes de clans et de cliques chez l'élite décisionnelle;

- transitivité de l'information utile aux décideurs;

- capacité d'apprentissage collectif.

Bien que ces facteurs relèvent plus du qualitatif que du quantitatif, chaque territoire local ou régional les possède à un certain degré. L'observation attentive selon une approche ethnologique peut nous révéler ces qualités de la culture territoriale. L'objet est à cet effet sûrement moins concerné par la mesure précise que par l'identification des divers facteurs sous leur angle dynamique en pointant des facteurs de progrès, vers un gain qualitatif global.

À cet égard, Bassand (1990) démontre fort bien que les processus identitaires s'avèrent un élément essentiel de base de cette évolution qualitative. On en déduit dès lors que la transformation d'un territoire local ou régional en milieu innovateur est fortement liée à la présence plus ou moins importante, chez les individus et les organisations, de l'identité au territoire en question. Nous la considérons, en conséquence, telle la première réquisition dans l'organisation générale ou la planification d'un milieu innovateur. 
Ainsi, le découpage territorial d'un éventuel milieu innovateur à planifier doit s'inspirer de ce principe d'identité territoriale. Il existe, à ce sujet, des symboles d'appartenance territoriale reliés à la géographie, à l'histoire, aux ressources naturelles, à l'architecture, aux activités sociales, culturelles et économiques, etc. qu'il ne faut certes pas négliger. Toutefois, l'aire de marché (de gestion) des diverses organisations, notamment celles reliées fonctionnellement aux facteurs inclus dans le modèle octogonal illustré ci-dessus, devient un critère très important pour considérer concrètement le découpage en fonction de l'identité territoriale.

\section{Identification et positionnement des acteurs du milieu}

Par ailleurs, la notion de culture territoriale nous oblige aussi à focaliser sur la dynamique entre les acteurs (décideurs des organisations). Puisque le caractère d'évolution est essentiel à la qualité d'innovation, c'est le processus organisationnel ou structurant qui s'avère important pour le milieu innovateur et non pas une structure institutionnelle quelconque, établie à priori ou à posteriori. Blakely (1987) définit en ce sens le développement local et régional tel un processus culturel continu de construction institutionnelle du territoire. Les décideurs des organisations sont ainsi directement interpellés comme acteurs du processus de création continue d'un milieu innovateur sur le territoire identifié. Ceux-ci ${ }^{11}$ sont à priori fort nombreux et fort divers. Il s'agit de les identifier, de les qualifier et de les positionner dans l'environnement organisationnel du territoire en question. Pour ce faire, le modèle proposé par Friedmann (1992) nous apparaît tout à fait approprié. Cet auteur différencie les quatre grandes sphères de la pratique sociale:

- l'État: appareil législatif, exécutif et judiciaire;

- la société civile : citoyens, propriétaires, ménages, familles, clans, ethnies, classes sociales, etc.;

- l'économie incorporée: corporations privées et leurs intérêts collectifs;

- le communautaire: mouvements sociaux et organisations politiques indépendantes.

11. On peut aussi y retrouver des décideurs potentiels, c'est-à-dire des individus qui ne représentent pas une organisation, mais qui sont en quête de responsabilités en ce sens. 
Ces quatre grandes sphères d'activités ne permettent pas une classification tout à fait tranchée et précise dans un territoire, des organisations qui peuvent éventuellement contribuer à la création d'un milieu innovateur. Plusieurs de celles-ci appartiennent à deux sphères à la fois alors que certaines relèvent de trois sphères. Ainsi, nos sphères s'entrecoupent les unes avec les autres (schéma 3 ).

SCHÉma 3

\section{Les acteurs du milieu innovateur}

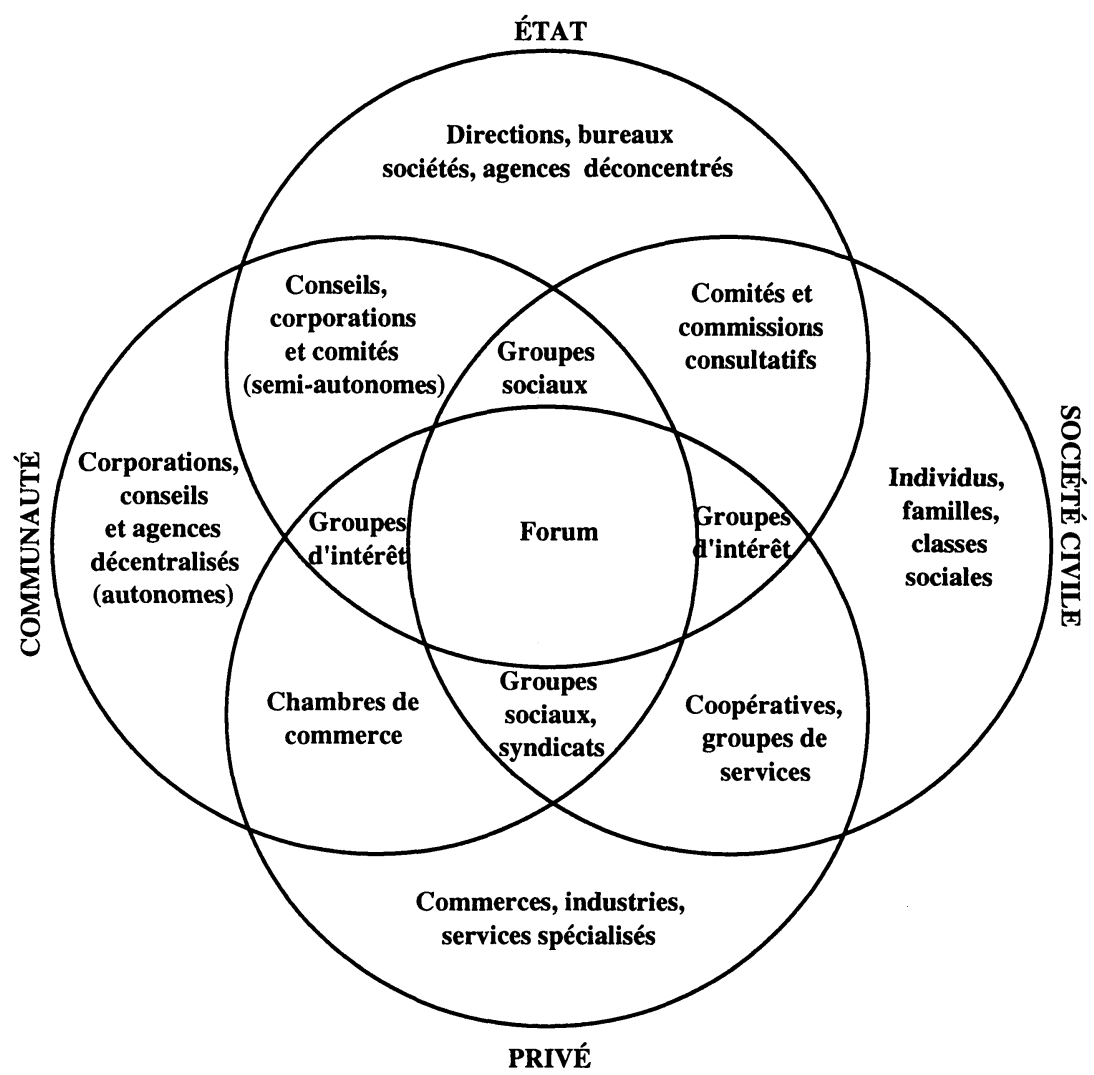

L'application de ce modèle général sur un territoire local ou régional délimité nécessite évidemment une certaine quantité d'information sur chaque organisation afin d'être en mesure de la localiser dans la bonne zone du schéma quadrisphérique. Les organisations unidimensionnelles sont relativement faciles à positionner. Cependant, plusieurs organisations bidimensionnelles ou tridimensionnelles nécessitent un peu plus d'attention. À cet égard, il faut, à notre sens, connaître certaines caractéristiques minimales telles que: 
- le statut de l'organisation;

- la territorialité (territoire de gestion; aire de marché);

- la structure organisationnelle interne;

- les sources de financement;

- la mission et les fonctions exercées;

- les objectifs et les orientations;

- le processus décisionnel et l'autonomie.

À partir de ces renseignements que l'on recueillera sur chaque organisation, il devient possible de localiser chacune dans notre modèle d'analyse. Pour ce faire, il s'agit d'inventorier les organisations privées, publiques et collectives présentes, d'analyser les caractéristiques de leur profil et de les positionner au bon endroit dans le modèle. Le schéma 3 illustre notre effort de localisation des organisations dans les territoires régionaux du Québec.

Outre le recensement complet des organisations qui utilisent dans leur gestion le territoire investigué, cette démarche de positionnement et de classification nous permet de considérer les fonctions exercées par rapport aux éléments de notre modèle octogonal des milieux innovateurs présenté au schéma 2. Ainsi, en connaissant mieux les organisations présentes sur le territoire, on pourra identifier et sensibiliser celles qui sont les plus aptes à collaborer étroitement au processus collectif de création d'un milieu innovateur.

\section{6. Établissement d'un forum territorial de réflexion collective}

L'espace de croisement entre les quatre sphères du modèle de Friedmann semble de toute évidence un lieu privilégié d'occupation puisqu'il touche les quatre sphères de la pratique sociale, c'est-à-dire tous les décideurs du territoire. À cet égard, le mode d'occupation éventuelle de ce carrefour territorial doit être spécialement adapté à la complexité des intérêts en jeu, à l'autonomie des multiples décideurs, au respect des structures institutionnelles existantes et à l'état de la culture organisationnelle sur le territoire.

En considérant les organisations privées, publiques et collectives déjà en place, dont le degré d'autonomie rend fort difficile, en principe, l'introduction d'une planification dirigiste d'un milieu innovateur sur le territoire sélectionné, nous préconisons la mise en place d'un forum territorial afin d'enclencher et d'animer un processus d'intelligence collective. Un grand nombre de territoires 
locaux et microrégionaux chez la plupart des pays occidentaux ont déjà expérimenté avec succès, ces dernières années, un tel lieu neutre capable d'engendrer le dialogue et les échanges entre les divers décideurs (OCDE, 1985...1993). Dans le cadre particulier des petites régions du Québec (Proulx, 1991), nous avons constaté que la mise en œuvre d'un tel forum permet en fait de doter le territoire d'un cerveau et d'un processus décisionnel collectifs unissant ainsi rationalité et démocratie dans une vision globale du devenir communautaire. Il faut préciser que le forum territorial préconisé n'est pas une structure lourde, mais bien un simple lieu de mise en communication des décideurs. Ainsi, tous peuvent s'exprimer librement, se renseigner sur les enjeux collectifs, défendre des opinions et des principes ainsi que s'engager sur des priorités collectives. Des initiatives d'organisation territoriale peuvent y être pilotées, notamment les étapes d'une procédure de planification d'un milieu innovateur.

\section{Définition d'une vocation de milieu innovateur}

Par l'animation d'un forum territorial capable de mobiliser tous les décideurs intéressés, sans distinction, la collectivité en question lance inévitablement un débat de fond sur ses valeurs, finalités et buts communautaires. L'intelligence supérieure qui est créée par ce processus doit idéalement être en mesure:

- d'identifier les valeurs progressistes à l'aube du XXI" siècle;

- d'identifier les grands enjeux sociaux, culturels et économiques actuels;

- d'élaborer des dossiers d'information sur des thèmes pertinents ;

- d'explorer des scénarios futurs possibles et désirables pour le territoire;

- de débattre systématiquement sur des éléments de choix de société territoriale;

- d'explorer la faisabilité d'éventuelles actions structurantes d'un milieu innovateur.

Un tel processus de réflexion collective systématique sur le devenir du territoire doit nécessairement conduire à la détermination d'une vocation pour l'éventuel milieu innovateur désiré par la collectivité. La littérature nous enseigne à cet égard qu'une telle vocation sélectionnée est généralement sectorielle (textile, micro-électronique, culture, agro-alimentaire, tourisme, etc.) mais peut aussi être intersectorielle sur des enjeux porteurs tels que: le secteur maritime, le secteur rural, l'exportation, les métiers d'art, la diplomatie, la haute technologie et le commercial, etc. La définition de la vocation du milieu doit préciser les grands objectifs à atteindre à court, moyen et long terme. 


\section{Mise en œuvre d'un tableau de bord territorial}

Bien que plusieurs de ces composantes apparaissent inévitablement dans le cadre de l'établissement de la vocation du milieu innovateur, la confection d'un portrait global de la situation territoriale (bilan, diagnostic, profil) représente une étape spécifique dans la planification d'un milieu innovateur. Une fois conçu, un tel portrait doit être enrichi et réactualisé constamment afin de prendre la forme concrète d'un véritable tableau de bord territorial. Cet instrument de traitement et de stockage de données permet d'éclairer les divers décideurs par des indicateurs pertinents ${ }^{12}$ et fiables sur la base environnementale et la base socioéconomique dont les principales dimensions sont les suivantes:

- éléments de l'environnement naturel;

- démographie et ressources humaines;

- environnement construit (cadre bâti);

- caractéristiques économiques générales;

- problématiques et analyses sectorielles (forces concurrentielles);

- système institutionnel endogène et exogène;

- facteurs communautaires.

Chacune de ces dimensions renferme un certain nombre de composantes qui doivent être idéalement mesurées par une batterie d'indicateurs. Le tableau complet ainsi confectionné et réactualisé devient une banque de données accessible à tous les décideurs du milieu innovateur en devenir. Si l'on considère l'incertitude dans laquelle baignent les décideurs qui manquent d'information pertinente, l'utilité stratégique d'un tel instrument pour le territoire en question apparaît sans équivoque.

\section{9. Élaboration de stratégies de milieu innovateur}

À partir du moment où le territoire s'est défini une vocation et qu'il dispose de toute l'information sur le portrait global de la situation, il s'agit maintenant de se doter de stratégies capables d'orienter le devenir d'un véritable milieu innovateur. En effet, les stratégies permettent de rassembler, de coordonner et d'encadrer les diverses actions fragmentées des multiples organisations vers des buts et finalités collectifs à atteindre.

12. Données qualitatives et quantitatives sur les ressources, les besoins, les problèmes, les faiblesses, les opportunités et les contraintes. 
Les champs stratégiques d'intervention sur le territoire à planifier sont à priori fort nombreux surtout si nous utilisons la division traditionnelle par secteurs (santé, éducation, loisirs, agriculture, acier, etc.). Dans le cas qui nous concerne, le modèle octogonal présenté plus haut indique huit grands thèmes stratégiques correspondant aux grands enjeux reliés à la capacité d'innovation. Pour chacun des thèmes, il devient essentiel d'organiser une table de réflexion collective concernée par l'élaboration éventuelle d'une stratégie commune aux divers intervenants dans le champ en question.

À cet égard, les tables de réflexion stratégique peuvent avoir recours à plusieurs sources d'information pour enrichir le débat:

- grandes tendances anticipées à moyen terme;

- composantes des théories du développement;

- vocation, finalités et buts du territoire;

- portrait de la situation territoriale et problématiques sectorielles;

- contexte et conjonctures internationales;

- banque d'outils disponibles pour le développement;

- banque de projets d'actions potentiellement faisables dans le milieu;

- stratégies, politiques et programmes du secteur public (tous les échelons);

- stratégies des organisations privées;

- stratégies et politiques des organisations collectives;

- stratégies mises en œuvre sur d'autres territoires similaires;

- stratégies antérieures mises en œuvre sur le territoire.

Sur la base de cette information stratégique, chaque table doit se doter d'une problématique faisant ressortir les forces, les faiblesses, les opportunités, les contraintes, les besoins sur le thème débattu. Ensuite, les intervenants se penchent sur la conception de grands objectifs tels que: diversification, assainissement, accroissement, valorisation, aménagement, élimination, protection, structuration, réduction, concentration, dispersion, conservation, etc. Ces objectifs doivent être mesurables au triple plan des extrants anticipés (emplois, investissements, production, valeur ajoutée, effets induits, etc.), des intrants nécessaires (ressources naturelles, humaines, construites et financières) et des intrants disponibles pour la réalisation concrète. Puisque les ressources sont limitées, la réduction, l'accroissement ou l'élimination d'un objectif provoque l'ajustement des intrants disponibles pour les autres objectifs. Ainsi, les 
multiples combinaisons intrants-extrants deviennent les arguments à considérer dans l'évaluation comparative de la faisabilité des différentes options stratégiques proposées par les décideurs. Comparaison qui doit évidemment se situer vis-à-vis les finalités, les buts et grands objectifs reliés à la vocation du milieu. Pour chaque table thématique, la nécessaire priorisation d'une ou de quelques stratégies par consensus entre les intervenants possède ainsi une base rigoureuse de critères rationnels.

\section{Montage d'actions structurantes pour le milieu innovateur}

Parmi les paramètres de l'évaluation des options stratégiques visant la transformation d'un territoire en véritable milieu innovateur, il faut nécessairement considérer les opportunités d'actions. Ainsi, l'analyse de la préfaisabilité des éventuelles actions structurantes (production, équipements, infrastructures, services, etc.), devient essentielle.

Pour ce faire, il s'agit de monter le dossier d'opportunité, c'est-à-dire préparer le document qui décrit non seulement l'action potentielle, mais aussi les diverses composantes (coûts, bénéfices, impacts, contraintes) de la réalisation. À cet égard, la procédure classique prévoit trois grands éléments :

- identification et mesure des intrants nécessaires en termes de ressources ;

- identification et mesure des extrants anticipés au plan de l'effet structurant ;

- établissement de la faisabilité multicritère (technique, environnementale, administrative, financière, sociale, politique, économique...) de l'opportunité.

Avec ce matériel, le responsable de chaque dossier pourra constituer la présentation de l'opportunité ainsi que l'argumentation systématique afin de confronter le projet aux diverses étapes du cheminement vers l'engagement des décideurs dans sa réalisation concrète.

\section{Recherche de tactiques d'exécution des actions}

On définit généralement la tactique comme l'art d'utiliser les moyens disponibles afin d'exécuter des actions en fonction des stratégies retenues. Il s'agit dès lors d'utiliser de manière opportune (ruse, rapidité, initiatives spontanées, démonstration, etc.) les conditions conjoncturelles qui se présentent face à 
l'opportunité d'action. Dans leur désir d'opérationnaliser les stratégies en actions concrètes visant la transformation d'un territoire en milieu innovateur, les tacticiens doivent considérer systématiquement certaines fonctions et tâches essentielles:

1. Confection d'un plan articulé de développement du milieu innovateur

- valeurs, buts, finalités et vocation du milieu;

- portrait global de la situation du territoire (synthèse);

- orientations stratégiques élaborées et priorisées;

- dossiers d'actions structurantes pour le milieu.

2. Inventaire des moyens disponibles pour le milieu

- marché privé (investissements, prêts, actions...);

- programmes du secteur public à tous les échelons;

- secteur communautaire;

- fonds populaire;

- organisations internationales d'aide au développement.

3. Considérations des conditions du champ

- terrain (décideurs en place, population, climat social, conjoncture économique, etc.);

- ambiance (pouvoir, compétition, conflits, partisanerie, alliances traditionnelles, etc.);

- solidarité (sensibilisation, mobilisation, consensus collectif, etc.);

- contexte (démarches, manifestation, pressions, action directe, médias, etc.).

4. Présentation des projets de manière systématique

- désignation formelle du porteur de dossier;

- dossier complet de la faisabilité;

- décideurs engagés formellement;

- marketing;

- échéancier de réalisation.

Il va sans dire que les tacticiens travaillent sur des tâches spécifiques, limitées et à court terme: il s'agit d'exécuter des actions. Le cheminement 
étant à cet égard souvent tortueux, la tactique fait vraiment appel aux qualités des ressources humaines engagées sur les dossiers d'actions structurantes du territoire en milieu innovateur.

\section{Conclusion}

Après avoir exposé le modèle théorique des milieux innovateurs, nous venons de proposer une démarche articulée capable d'engendrer le processus de leur création dans les territoires locaux et régionaux qui le désirent. Pour ce faire, ceux-ci doivent impérativement posséder les caractéristiques nécessaires au point de vue de l'identité collective, de la culture territoriale et de la dynamique entre les organisations. Pilotée par un cerveau collectif prenant assise dans un forum territorial, la procédure offerte intègre les récents apports théoriques et empiriques de la planification territoriale et prévoit l'utilisation des techniques modernes pour son opérationnalisation. Ainsi, la définition de la vocation spécifique chez l'éventuel milieu innovateur, la mise en œuvre d'un tableau de bord, l'élaboration de stratégies, le montage d'actions structurantes, ainsi que la recherche de tactiques pour exécuter ces dernières deviennent, dans leurs diverses composantes, les éléments organisationnels pour l'application concrète du concept de milieu innovateur dans un territoire local ou régional.

Il va sans dire que tous les territoires qui utilisent cette procédure n'atteindront pas le même succès dans leur transformation systématique en milieu innovateur, car tous n'ont pas les mêmes ressources, les mêmes besoins, les mêmes opportunités et les mêmes contraintes au départ de l'exercice. Cependant, la dotation d'une démarche collective et d'une procédure articulée de planification territoriale favorise l'émergence d'une synergie qui génère non seulement des espoirs, mais aussi de véritables activités dans la plupart des cas. À cet égard, plusieurs auteurs de la littérature attachent plus d'importance à l'une ou l'autre des étapes de la procédure, notamment l'élaboration des stratégies appropriées. De notre côté, nous avançons que c'est la qualité de la démarche collective globale qui compte. La planification doit être mise en œuvre d'une manière continue, c'est-à-dire qu'il faut certes définir une vocation de long terme, mais aussi réactualiser et réévaluer les options (stratégies-actions) régulièrement selon la variation des indicateurs du tableau de bord territorial. C'est dans un tel processus collectif continu d'apprentissage que réside, à notre avis, le secret des futurs milieux innovateurs. 


\section{Bibliographie}

AYDALOT, P. (1984), «Technologies nouvelles et développement territorial », Rapport préliminaire et projet de recherche, Groupe technologies nouvelles et espace, La Sorbonne, Paris.

AYDALOT, P. (1986), Milieux innovateurs en Europe, Paris, éd. Groupe de recherche européen sur les milieux innovateurs.

Aydalot, P. et D. KeEBle (1988), High Technology Industry and Innovative Environments, Londres, Routledge.

BerThalanfFy, L. (1950), "The theory of open system in physics and biology ", Science, vol. 3.

BASSAND, M. (1990), Culture et régions en Europe, Lausanne, Presses polytechniques romandes.

BASSAND, M. et al. (1985), Innovation et changement social, Lausane, Presses polytechniques romandes.

BENKO, G et A. LIPIETZ (1992), Les régions qui gagnent, Paris, PUF, p. 35-56.

BECATTINI, G. (1992), «Le district marshallien: une notion socio-économique», dans Benko et Lipietz, op. cit.

Blakely, E.J. (1987), Planning Local Economic Development, Londres, Sage Publications.

CAmagni, R. (1992), Innovation Networks - Spatial Perspectives, Londres, Belhaven Press.

CHICHA, J. et al. (1985), La PME dans un monde en mutation, Sainte-Foy, Presses de l'Université du Québec.

FAludi, A. (1987), A Decision-Centred View of Environmental Planning, Londres, Pergamon Press.

FriedmanN, J. (1992), Empowerment, Cambridge, USA, Blackwell Publishers.

FriedmanN, J. (1987), Planning in the Public Domain: From Knowledge to Action, New-Jersey, Princeton University Press.

GAGNON, C. et J.L. KLEIN (1991), «Le partenariat dans le développement local», Cahiers de Géographie du Québec, vol. 35, n 95, p. 239-256.

JESSOP, J. et C.M. WEAVER (1987), «La coopération communautaire: une stratégie pour le développement local», Revue Internationale d'Action Communautaire, vol. 13, $\mathrm{n}^{\circ} 53$.

MAILlat, D. (1992), «Milieux et dynamique territoriale de l'innovation», Revue Canadienne de Science Régionale, vol. $\mathrm{XV}, \mathrm{n}^{\circ} 3$.

MAILlAT, D. et J.C. PERRIN (1992), Entreprises innovatrices et développement territorial, Neuchâtel, éd. GREMI, EDES. 
MaILlat, D. et al. (1993), «Réseaux d'innovation et milieux innovateurs : un pari pour le développement régional», Neuchâtel, Groupe de recherche européen sur les milieux innovateurs, EDES.

Marshall, A. (1890), Principles of Economic, Londres, Macmillan.

MARSHALl, A. (1919), Industry and Trade, Londres, Macmillan.

MUlfORD, R. (1983), Interorganizational Relations, New York, Human Sciences Press.

OCDE (1982), «Programme ILE : étude préliminaire des activités», Paris, Publication de l'Organisation de coopération et de développement économique.

OCDE (1985...1993), «Les Cahiers des ILES », Paris, Publications de l'Organisation de coopération de développement économique dans le cadre du Programme ILE.

PERRIN, J.C. (1983), « Contribution à une théorie de la planification décentralisée », dans B. Planque (éd.), Le développement décentralisé (éd.), Paris, GRAL, LITEC.

PERRIN, J.C. (1992), «Pour une révision de la science régionale : l'approche en termes de milieu », Revue Canadienne de Science Régionale, vol. $\mathrm{XV}, \mathrm{n}^{\circ} 3$.

PiORE, M. et C. SABEL (1984), The Second Industrial Divide: Possibilities for Prosperity, New York, Basic Books.

PlANQUE, B. (1988), «La PME innovatrice: quel est le rôle du milieu local », Revue Internationale $P M E$, vol. 1, $\mathrm{n}^{\circ}$ 2, p. 177-192.

PlANQue, B. (1992), «Les milieux innovateurs en PACA, France », Revue Canadienne de Science Régionale, vol. $\mathrm{XV}, \mathrm{n}^{\circ} 3$.

ProulX, M.U. (1989), «Activité résillière et organisation économique de l'espace », Revue d'Economie Régionale et Urbaine, $\mathrm{n}^{\circ} 3$, p. 575-600.

ProulX, M.U. (1991), "Réseaux utilitaires spatialisés et dynamique économique», Revue Canadienne de Science Régionale, vol. XIV, $\mathrm{n}^{\circ} 1$, p. 73-92.

RATTI, R. (1992), Innovation technologique et développement régional, éd. L.R.E., D.P.L.M.

RCSR (1992), Numéro thématique de la Revue Canadienne de Science Régionale sur la «Création de milieux innovateurs », vol. XV, $\mathrm{n}^{\circ} 3$.

RERU (1991), Numéro spécial de la Revue d'Économie Régionale et Urbaine, nos 3-4.

RIPME (1989), Numéro spécial de la Revue Internationale P.M.E., vol. 2, nos 2-3.

STOHR, W.B. (1985), «Le rôle stratégique de la concertation», dans M. Boisvert et P. Hamel (éd.), Redéploiement industriel et planification régionale, Université de Montréal.

STOHR, W.B. et D.R.F. TAYLOR (1981), Development from Above or Below, Londres, John Wiley \& Sons.

Weaver, C.M. (1984), Regional Development and the Local Community, New York, John Wiley \& Sons. 